\title{
artigo
}

Almeida, A.; Almeida, A.; Sousa, M.P.L.; Liberato, L.C.; Brasil, M.Y.O.; Lopes da Silva, C.R.;

O suicídio como um problema de saúde pública

\section{O suicídio como um problema de saúde pública}

\author{
Suicide as a public health problem \\ El suicidio como problema de salud pública
}

\begin{abstract}
RESUMO
Objetivo: Analisar e trazer uma reflexão sobre os altos índices de óbitos por suicídio. Método: Trata-se de uma revisão integrativa da literatura. A busca dos artigos foi realizada entre os meses de setembro e outubro de 2020. Foram utilizados como critérios de inclusão: artigos disponiveis em português e inglês, publicados na integra de forma gratuita entre os anos de 2017 e 2020, sendo exclusos os artigos repetidos, inconclusivos, relatos de experiencia, estudos reflexivos e estudos não pertinentes à temática. Resultados: Foram selecionados 6 artigos para elaboração da revisão. Evidenciou-se que apesar de ser um tema bastante polêmico e relevante, ainda existem tabus que dificultam na prevenção para lidar com esse evento. Conclusão: Por fim, torna-se necessário fortalecer a criação de estratégias para o manejo de pessoas em risco com o objetivo de reduzir a incidência de óbitos causadas por esse ato.
\end{abstract}

DESCRITORES: Suicídio. Saúde Pública. Preconceito. Ideação suicída.

\section{ABSTRACT}

Objective: To analyze and reflect on the high rates of deaths by suicide. Method: This is an integrative literature review. The search for articles was carried out between the months of September and October 2020. The following inclusion criteria were used: articles available in Portuguese and English, published in full free of charge between the years 2017 and 2020, with repeated articles being excluded, inconclusive, experience reports, reflective studies and studies not relevant to the theme. Results: Six articles were selected to prepare the review. It became evident that despite being a very controversial and relevant topic, there are still taboos that hinder prevention in dealing with this event. Conclusion: Finally, it becomes necessary to strengthen the creation of strategies for the management of people at risk in order to reduce the incidence of deaths caused by this act.

DESCRIPTORS: Suicide. Public health. Preconception. Suicidal ideation

\section{RESUMEN}

Objetivo: Analizar y reflexionar sobre las altas tasas de muerte por suicidio. Método: Se trata de una revisión bibliográfica integradora. La búsqueda de artículos se realizó entre los meses de septiembre y octubre de 2020. Se utilizaron los siguientes criterios de inclusión: artículos disponibles en portugués e inglés, publicados íntegramente de forma gratuita entre los años 2017 y 2020, excluyéndose los artículos repetidos, inconclusos, informes de experiencias, estudios reflexivos y estudios no relevantes al tema. Resultados: Se seleccionaron seis artículos para preparar la revisión. Se hizo evidente que a pesar de ser un tema muy controvertido y relevante, aún existen tabúes que dificultan la prevención para enfrentar este evento. Conclusión: Finalmente, se hace necesario fortalecer la creación de estrategias para el manejo de personas en riesgo con el fin de reducir la incidencia de muertes por este acto.

DESCRIPTORES: Suicidio. Salud pública. Preconcepción. Ideación suicida.

RECEBIDO EM: 16/11/2020 APROVADO EM: 15/12/2020

\author{
Alanny de Almeida \\ Discente do Curso de Enfermagem no Centro Universitário de Juazeiro do Norte - UNIJUAZEIRO \\ ORCID: 0000-0002-8216-2139
}

\section{Amanda de Almeida}

Discente do Curso de Enfermagem no Centro Universitário de Juazeiro do Norte - UNIJUAZEIRO

ORCID: 0000-0002-6204-4506

\section{Maria Paloma Lima Sousa}

Discente do Curso de Enfermagem no Centro Universitário de Juazeiro do Norte - UNIJUAZEIRO

ORCID: 0000-0002-3029-0307 


\section{Ludmila Cavalcante Liberato}

Discente do Curso de Enfermagem no Centro Universitário de Juazeiro do Norte - UNIJUAZEIRO

ORCID: 0000-0001-9422-4328

\section{Maria Yngrid Oliveira Brasil}

Discente do Curso de Enfermagem no Centro Universitário de Juazeiro do Norte - UNIJUAZEIRO

ORCID: 0000-0001-6816-3787

\section{Cícero Rafael Lopes da Silva}

Especialista em Enfermagem Dermatológica. Docente do curso de Bacharelado em Enfermagem no Centro Universitário de Juazeiro do Norte - UNIJUAZEIRO

ORCID: 0000-0001-8819-5380

\section{INTRODUÇÃO}

A morte sempre intrigou e espantou a humanidade, mesmo sendo um marcador social do qual nenhum humano será poupado. No que se refere morte voluntária, como o suicídio, intriga ainda mais, de forma que poderia ter sido adiada ou evitada. O suicídio é uma ocorrência social mundial que constitui um grave problema de saúde pública, observado desde a antiguidade e acontece em todas as culturas e gêneros ${ }^{1,2}$.

O suicídio é entendido como um fenômeno multifatorial e multidimensional em consequência da relação complexa entre diversos fatores, como ambientais, fisiológicos, biológicos, genéticos e sociais, entretanto ainda é considerado um tema com bastantes preconceitos e tabus em muitas sociedades ${ }^{3}$.

Segundo a Organização Mundial da Saúde (OMS) ${ }^{4}$, o suicídio baseia-se no ato intencional para acabar com a própria vida. É uma prioridade de saúde pública, apresentando-se como a terceira causa de morte em todo o mundo. Nos dias de hoje, a cada 40 segundos uma pessoa comete suicídio, e para cada suicídio houve entre 10 e 20 tentativas. Dados mostram que cerca de 800 mil pessoas tiram a sua própria vida todos os anos e um número ainda maior de indivíduos tentam suicídio, tornando a segunda principal causa de morte entre jovens com idade de 15 a 29 anos.

Entre os anos de 2007 e 2016, foram registrados no Sistema de Informações sobre Mortalidade (SIM) 106.374 óbitos por suicídio. O meio mais utilizado para colocar um fim na própria vida é por into- xicação exógena. No ano de 2016, A taxa média nacional de suicídio foi de 5,8 óbitos por 100 mil habitantes, com a notificação de 11.433 mortes por essa causa, 79\% dos suicídios ocorreram em países de baixa e média renda ${ }^{5}$.

Ainda considerado um evento cheio de enigmas pela sociedade, busca-se respostas para compreendê-lo, preveni-lo, sensibilizar a população e sanar esse episódio, uma vez que os suicídios podem ser evitados em tempo oportuno, a partir de evidências e intervenções de baixo custo, sendo necessária uma ampla estratégia multisetorial. É um fenômeno onde todos estão vulneráveis, e vem acometendo todas as camadas sociais. Tal fato confirma que o suicídio não deve ser considerado uma patologia, mas sim um problema social ${ }^{6}$.

Mesmo diante de dados e do reconhecimento da sua gravidade e complexidade, o suicídio ainda se configura como um tema tabu, cheio de estigmas. Pode-se observar um aumento da comunicação sobre o assunto, mas ainda de forma restrita e cautelosa. Assim, o tabu impossibilita que o tema seja tratado de forma ampla pela sociedade, resultando em consequências negativas, uma vez que o aumento da consciência é uma das maneiras de preveni-lo ${ }^{7}$.

Assim, na tentativa de intervenções focada em indivíduos com ideações suicidas, em Brasília, no ano de 2015 foi criada a campanha "Setembro Amarelo" pelo Centro de Valorização da Vida (CVV), pelo Conselho Federal de Medicina (CFM) e pela Associação Brasileira de Psiquiatria (ABP). Criada com o intuito de levar conhecimento a população sobre as formas de prevenção ao suicídio e alertar a todos a respeito desse problema, não apenas no Brasil, mas em todo o mundo ${ }^{8,9}$.

Diante desse contexto, objetiva-se analisar e trazer uma reflexão sobre os altos índices de óbitos por suicídio. Com a intenção de através dessa escrita sensibilizar a população da importância de abordar e compreender esse assunto, desfazendo-se de preconceitos e crenças que ainda estão presentes em nossa sociedade, visto que engana-se quem pensa que falar sobre o assunto irá estimular alguém a cometer tal ato, e mostrando assim, a necessidade da intervenção.

Diante desse contexto e da necessidade do conhecimento da problemática, o estudo tem como questão norteadora: qual a visão em relação ao suicídio nos dias atuais?

\section{MÉTODO}

Trata-se de uma revisão integrativa de literatura, metodologia que consiste na elaboração de uma análise extensa da literatura, cooperando assim para discussões sobre métodos e resultados de pesquisa, bem como reflexões sobre a realização de novos estudos. Este método de pesquisa tem como objetivo inicial obter uma compreensão maior sobre o tema investigado baseando-se em estudos anteriores ${ }^{10}$.

A busca dos artigos foi realizada entre os meses de setembro e outubro de 2020, nas bases de dados Scientific Eletronic Library Online (SciELO) e Biblioteca Virtual em Saúde (BVS) com artigos da Medical Literature Analysis and Retrieval System Online (MEDLINE) e Literatura Latino-americana e do Caribe em Ciências da Saúde (LILACS). Foram utilizados 


\section{artigo}

Almeida, A.; Almeida, A.; Sousa, M.P.L.; Liberato, L.C.; Brasil, M.Y.O.; Lopes da Silva, C.R.

O suicídio como um problema de saúde pública

como critérios de inclusão: artigos disponíveis em português e inglês, publicados na integra de forma gratuita entre os anos de 2017 e 2020, sendo exclusos os artigos repetidos, inconclusivos, os relatos de experiencia, estudos reflexivos e estudos não pertinentes à temática.

A preparação desta pesquisa originou-se com a consulta aos Descritores em Ciências da Saúde (DeCS) e no Medical Subject Headings $(\mathrm{MeSH})$. Foram, portanto, utilizados os seguintes descritores em português e inglês, respectivamente: "Suicídio" / "Suicide", "Saúde Pública" / "Public Health", "Preconceito" / "Prejudice" e/ou "Ideação suicida" / "Suicidal ideation". Sendo utilizado também o operador booleano AND para cruzamento dos termos.

\section{FLUXOGRAMA 1: Processo de seleção e inclusão dos artigos}

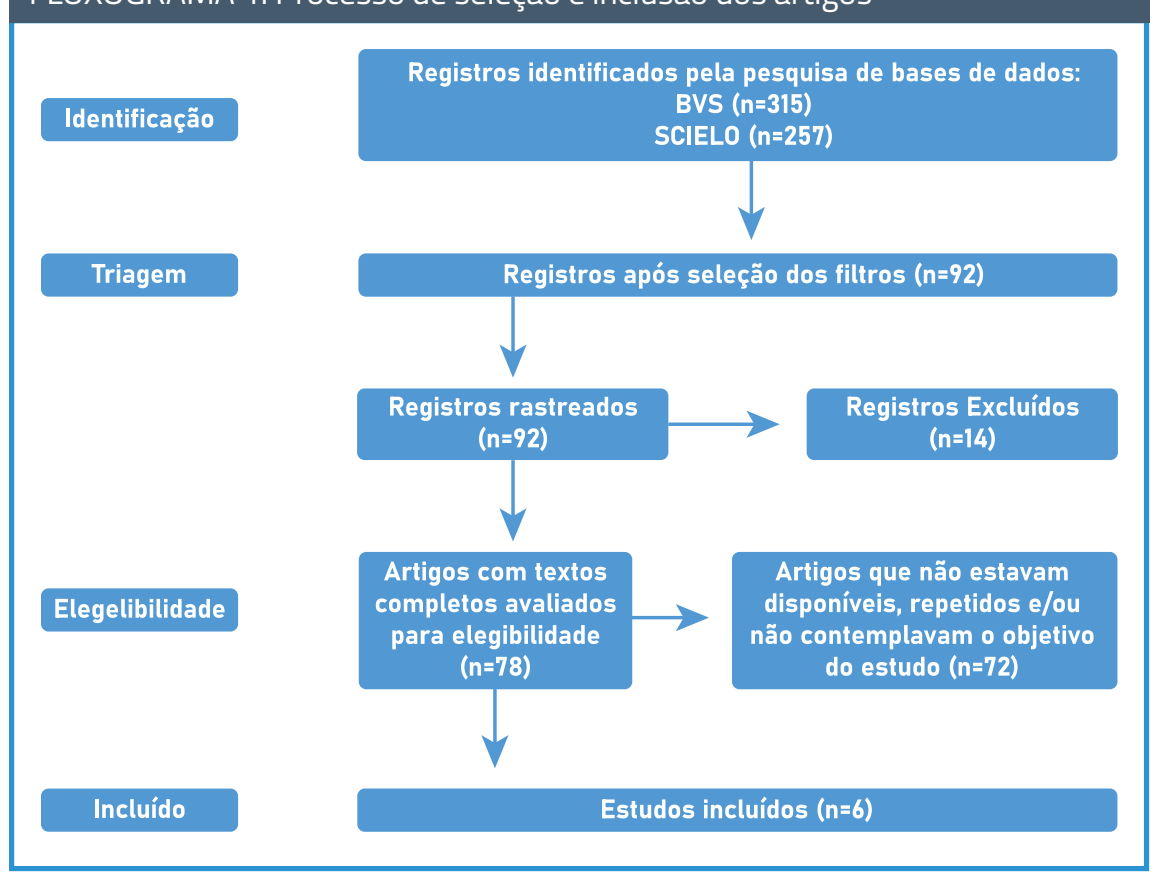

FONTE: PRISMA 2009 Flow Diagram

\section{Tabela 1: Síntese dos estudos inclusos na pesquisa.}

Periódico/Ano

Revista Sociedade e Estado - Volume 35, Número 1, Janeiro/ Abril 2020

Revista Psicologia em Foco, Frederico Westphalen, v. 11, n. 16, p. 19-32, nov. 2019

Saúde Soc. São Paulo, v.27, n.1, p.185-200, 2018
Título

A desesperança do jovem e o suicídio como solução e seu impacto na Saúde Pública

Aproximações e distanciamentos ao suicídio: analisadores de um serviço de atenção psicossocial
$\mathrm{Na}$ primeira junção foram encontrados 572 artigos, referindo-se a 257 na SciELO e 315 na BVS. Na segunda junção após a escolha dos filtros artigos entre os anos 2017 e 2020, No idioma português e inglês, totalizaram 92 artigos para análise e seleção final, desses apenas 78 estavam disponíveis, 23 não eram originais, 8 eram repetidos e 41 não contemplavam o objetivo do estudo, restando 6 artigos para compor o estudo. Utilizou-se o roteiro proposto na metodologia do Preferred Reporting Items for Systematic Reviws and Meta-Analyses (PRISMA) ${ }^{11}$ para o desenvolvimento do estudo de modo a organizar os artigos selecionados e ocasionar um melhor direcionamento dos dados obtidos.

\section{RESULTADOS}

$\mathrm{O}$ artigo foi construído com base nos guidelines da metodologia do PRISMA. A seleção de artigos para composição da presente revisão integrativa foi organizada no Fluxograma 1, o qual seguiu as recomendações PRISMA.

Os 6 artigos selecionados foram organizados na tabela 1 , onde estão apresentados: periódico/ano, título, objetivo e principais resultados de cada um dos estudos, de forma sucinta.
Investigar a atenção ao suicídio de um serviço de atenção psicossocial em um município de São Paulo

\section{Principais Resultados}

avaliar a interdependência entre as complexas relações sociais da contemporaneidade, sua influência na construção identitária dos jovens e a morte intencional como saída para dores emocionais.

Analisar o suicídio na atualidade e seu impacto na saúde pública, considerando os fatores de risco e o luto "daqueles que ficam".

Ao considerar o suicídio como uma preocupação mundial, observa-se a realização de muitas ações para o seu enfrentamento e solução, porém ainda encontramos muitas dificuldades na prevenção e protocolos para lidar com esse evento.

O comportamento suicida é uma tragédia familiar e pessoal, e ainda um grave problema de saúde pública. Visto que compreender é bastante complexo no contexto que estamos inseridos, onde a morte é algo que aterroriza a sociedade.

Compreendido atualmente como um fenômeno multidimensional e multifatorial, o suicídio ainda é considerado um tema tabu em muitas sociedades. 
Tendências e controvérsias nas pesquisas em ciências sociais e saúde, 2020.

REVISTA M. 44 João Fernando Marcolan Rio de Janeiro, v. 4, n. 7, p. 31-44, jan./jun. 2019

Revista Eletrônica Acervo Saúde /

Electronic Journal

Collection Health, 2020
Desmistificando os muros do silêncio: tendências dos estudos sobre o fenômeno suicídio e o impacto na saúde pública

O comportamento suicida na realidade brasileira: aspectos epidemiológicos e da política de prevenção

Série temporal do suicídio no Brasil: o que mudou após o Setembro Amarelo?
Compreender as tendências dos estudos que analisam o suicídio, bem como desmistificar o silêncio que permeia a presente temática.

Analisar dados epidemiológicos sobre o comportamento suicida e a política de prevenção ao suicídio.

Analisar a prevalência das notificações de suicídio no Brasil antes e após o lançamento da Campanha Setembro Amarelo (CSA) pelo Ministério da Saúde.
Com dados epidemiológicos assustadores uma pessoa comete suicídio a cada 40 segundo; atingindo todas as faixas etárias e classes sociais, não tem como ignorar os dados, os fatos confirmam o grande desafio que estamos enfrentando.

Os fatores de risco relacionados ao comportamento suicida devem ser levados em conta em toda a sua extensão, do planejamento até o ato consumado. Vale ressaltar, que a tentativa de suicídio é um fator de risco para uma próxima tentativa.

A campanha Setembro Amarelo tem o intuito de levar conhecimento a população sobre suicídio e alerta-los a respeito desse problema, para que busquem ajuda e intervenções de profissionais qualificados.

FONTE: Próprios autores, 2020

Observa-se uma alta incidência de óbitos por suicídio e dificuldades na prevenção para lidar com esse evento. Além de ser uma tragédia pessoal e familiar, é um importante problema de saúde pública, onde todos estão vulneráveis a esse fenômeno. Sendo necessário falar sobre o assunto e afastar os preconceitos tabus presentes na sociedade.

\section{DISCUSSÂO}

As taxas de suicídio aumentam anualmente. No Brasil houve 183.484 mortes registradas por suicídio entre os anos de 1996-2016, apresentaram um aumento de 69,6\% de casos de suicídio nesse período, e representam um grave problema de saúde pública $^{12}$. Os números mesmo subestimados são assustadores, e alertam para a possibilidade desses números serem bem maiores, pois alguns suicídios são considerados e muitas vezes registrados como acidentes ou como morte de causa indeterminada.

Em relação à lesão autoprovocada, o banco de dados brasileiro do Ministério da Saúde, pelo Sistema de Informação de Agravos de Notificação (SINAN), e do Departamento de Informática do Sistema Único de Saúde (DATASUS), registrou, no período de 2009 a 2016, 186.891 notificações, sendo que 3.941 em 2009, 6.739 em 2010, 14.940 em 2011, 21.164 em 2012, 25.468 em 2013, 29.707 em 2014,

\section{Os números mesmo}

\section{subestimados}

\section{são assustadores,}

e alertam para a

possibilidade desses

números serem

bem maiores, pois

alguns suicídios

são considerados

e muitas vezes

registrados como

acidentes ou como

morte de causa

indeterminada.
39.464 em 2015, e 45.468 em $2016^{13}$. Embora o aumento no número de casos notificados chame a atenção, ainda falta um programa de vigilância ao comportamento suicida, visto que a tentativa de suicídio é um importante fator de risco para que o ato se repita.

Considerado um fenômeno complexo, de difícil compreensão e multifatorial, a análise dos fatores relacionados ao suicídio são essenciais e pode auxiliar profissionais de saúde a considerar o risco e traçar estratégias para reduzi-lo e evitar que o ato venha a ocorrer. Dentre os fatores de risco, podemos destacar transtornos mentais, isolamento social, questões psicológicas, condições clinicas incapacitantes e tentativas prévias de suicídio. As tentativas devem ser tratadas com seriedade e como sinal de alerta, visto que em cada suicídio consumado, houve pelo menos entre $10 \mathrm{e}$ 20 tentativas ${ }^{5}$.

Apesar do reconhecimento da gravidade e complexidade desse fenômeno, o suicídio ainda é tratado como um tema com bastante tabu e crenças errôneas, visto que isso dificulta falar abertamente sobre o assunto com a sociedade.

Torna-se necessário buscarmos um melhor entendimento que vá além dos preconceitos da sociedade, onde ainda se pensa que falar sobre suicídio é estimular a sua incidência. Mas longe disso, estratégias de saúde devem ser criadas e imple- 


\section{artigo}

Almeida, A.; Almeida, A.; Sousa, M.P.L.; Liberato, L.C.; Brasil, M.Y.O.; Lopes da Silva, C.R.

O suicídio como um problema de saúde pública

mentadas através do CVV, bem como do Setembro Amarelo, para que ocorra uma associação entre os setores e a sociedade, pra assim atuar na prevenção do suicídio e prestar uma assistência integral a população, isso em razão que o suicídio é uma questão social ${ }^{6}$.

Quando se analisa o suicídio, observa-se o quanto é complicado lidar com tal problemática, sendo que esse fenômeno, atualmente, mata mais que homicídios, problemas de saúde e acidentes de transito, sem contar com as falhas que encontramos no sistema, por exemplo, nem todas as ocorrências são registradas, tais informações seriam extremamente importantes no que se refere a prevenção, pois teríamos dados mais reais da situação ${ }^{1}$.

Desse modo, por se tratar de um evento complexo e multifacetado, as ações devem ser voltadas aos fatores de risco e de proteção, bem como o acolhimento adequado a população. Vale ressaltar a limitação da coleta de dados, uma vez que o sistema ainda é frágil e em construção, e com problemas em relação à subnotificação e omissão dos dados ${ }^{14}$.

Esse tema traz questionamentos e analises necessárias para definir estratégias de prevenção e de suporte tanto para a notificação quanto para a atenção em rede. É imprescindível lembrar a importância da sensibilização e qualificação de profissionais para atuarem frente a identificação e o acompanhamento de pessoas em risco ou com histórico de tentativa de suicídio, e também suas famílias. Importante que os profissionais tenham conhecimento a respeito das medidas de prevenção contra o suicídio e atuar na identificação precoce as pessoas que se encontram vulneráveis, assim procurando reduzir a incidência de óbitos por esta causa.

\section{CONSIDERAÇÕES FINIAIS}

Diante dos dados epidemiológicos apresentados na pesquisa, falar sobre suicídio torna-se relevante e atual, configurando a ser um problema de saúde pública que requer uma atenção maior e criação de estratégias para reduzir a sua incidência. Portarias, recomendações e leis são criadas, mas torna-se necessário ações integradas nas diferentes esferas socio psíquicas que envolvam toda a sociedade.

Por fim, cabe ressaltar que por ser um tema bastante polêmico, desafiador e com forte impacto, as pessoas que já apresentaram tentativas de suicídio não devem ser julgadas, e sim tratadas com empatia e amparo, com o intuito de fornecer fatores de proteção a essas pessoas. Fortalecendo a criação de estratégias para o manejo de pessoas em situaçóes de risco para o suicídio através da identificação de sinais de alerta, e a partir destes, o desenvolvimento de intervenções para minimizar este desfecho.

\section{REFERÊNCIAS}

1. Penso MA, Sena DPA. A desesperança do jovem e o suicídio como solução. Revista Sociedade e Estado - Volume 35, Número 1, Janeiro/Abril 2020

2. Gomes ER, Inglesias A, Constantinidis TC. Revisão Integrativa de Produções Científicas da Psicologia Sobre Comportamento Suicida. Revista Psicologia e Saúde, v. 11, n. 2, maio/ago. 2019, p. 35-53. ISSN: 2177-093X

3. Cescon LF, Capozzolo AA, Lima LC. Aproximações e distanciamentos ao suicídio: analisadores de um serviço de atenção psicossocial . Saúde Soc. São Paulo, v.27, n.1, p.185-200, 2018. DO 10.1590/S0104-12902018170376

4. ORGANIZAÇÃO MUNDIAL DA SAÚDE (OMS). Folha informativa sobre suicídio. Genebra: OMS, 2018.

5. BRASIL. Agencia Saúde. Novos dados reforçam a importância da prevenção do suicídio. 2018. Disponivel em: http://www. saude.gov.br/noticias/agencia-saude/44404-novos-dados-reforcam-a-importancia-da-prevencao-do-suicidio . Acesso em 23 de setembro de 2020.

6. Lóss JCS, Istoe RSC, Santos MFR. Desmistificando os muros do silêncio: tendências dos estudos sobre o fenômeno suicídio e o impacto na saúde pública. Tendências e controvérsias nas pesquisas em ciências sociais e saúde. Campos dos Goytacazes, RJ : Brasil Multicultural, 2020.

7. Teixeira SMO, Souza LEC, Viana LMM. O SUICÍDIO COMO QUESTÃO DE SAÚDE PÚBLICA. Rev Bras Promoç Saúde, Fortaleza, 31(3): 1-3, jul./set., 2018.
8. Oliveira MEC, Gomes KAL, Nóbrega WFS, Gusmão ECR, Santos RDS, Franklin RG. Série temporal do suicídio no Brasil: o que mudou após o Setembro Amarelo? Revista Eletrônica Acervo Saúde / Electronic Journal Collection Health, 2020. DOI: https:// doi.org/10.25248/reas.e3191.2020

9. Rambo M, Pezzi FAS. A dor do viver: o suicídio e seu impacto na saúde pública. Revista Psicologia em Foco, Frederico Westphalen, v. 11, n. 16, p. 19-32, nov. 2019.

10. Mendes KDS, Silveira RCCP, Galvão CM. Revisão integrativa: método de pesquisa para a incorporação de evidências na saúde e na enfermagem. Texto Contexto Enferm, Florianópolis, 2008 Out-Dez; 17(4): 758-64.

11. Moher D, Liberati A, Tetzlaff J, Altman DG, The PRISMA Group (2009). Preferred Reporting Items for Systematic Reviews and Meta-Analyses: The PRISMA Statement. PLoS Med 6(7): e1000097. doi:10.1371/journal.pmed1000097

12. Marcolan JF, Silva DA. O comportamento suicida na realidade brasileira: aspectos epidemiológicos e da política de prevenção. REVISTA M. Rio de Janeiro, v. 4, n. 7, p. 31-44, jan./jun. 2019

13. BRASIL. Ministério da Saúde. Secretaria de Vigilância em Saúde. Boletim Epidemiológico. Brasília. v. 48, n. 30, p. 1-14, 2017.

14. Rossi LM, Marcolino TQ, Speranza M, Cid MFB. Crise e saúde mental na adolescência: a história sob a ótica de quem vive. Cad. Saúde Pública 2019; 35(3):e00125018 